\title{
Terapêutica crónica em idosos numa Unidade de Saúde Familiar: análise da polimedicação e medicação potencialmente inapropriada
}

Márcia Mendonça Souto, ${ }^{1}$ Ana Filipa Pimentel ${ }^{2}$

\section{RESUMO}

Introdução: A melhoria dos cuidados de saúde e das condições socioeconómicas contribuíram para o aumento da longevidade da população, associando-se-lhe uma maior prevalência das doenças crónicas. A polimedicação, muitas vezes necessária nesta população, condiciona um aumento do risco da doença iatrogénica. As interações medicamentosas, bem como o uso de medicação potencialmente inapropriada a que o idoso fica exposto, pode resultar em consequências nefastas para a própria saúde. Objetivos: Este estudo pretendeu conhecer a terapêutica crónica nos idosos com idade igual ou superior a 75 anos, utentes de uma unidade de saúde familiar de Braga e, especificamente, pretendeu determinar a frequência de polimedicação, medicação potencialmente inapropriada e fatores predisponentes associados.

Resultados: Foram estudados 86 utentes, 96,5\% estavam a fazer toma, de forma crónica, de pelo menos um medicamento, $24 \%$ consumiam dois a quatro fármacos e $74,4 \%$ consumiam cinco ou mais fármacos na população estudada. Em $37,7 \%$ da amostra existia medicação potencialmente inapropriada segundo os Critérios de Beers, tendo em $90,7 \%$ dos casos grau de inadequação «elevado». Verificou-se associação, com significado estatístico, entre o número de fármacos e medicação potencialmente inapropriada. Estas duas variáveis relacionaram-se ainda, de forma estatisticamente significativa, com a «autoperceção da saúde», com «quem prepara a medicação» e com o «conhecimento da finalidade da medicação».

Discussão/Conclusão: Polimedicação e medicação potencialmente inapropriada são extremamente frequentes nos utentes, com idade igual ou superior a 75 anos, na população estudada. Os autores procuraram a partir dos dados obtidos ter uma compreensão do contexto socio-económico associado a maior risco, para ajudar a estabelecer uma estratégia de diminuição dos riscos de iatrogenia.

Palavras-chave: Polimedicação; Medicação potencialmente inapropriada; Critérios de Beers.

\section{INTRODUÇÃO}

O envelhecimento da população

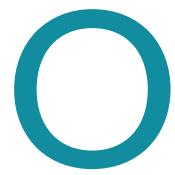
aumento da esperança média de vida em Portugal, nas últimas décadas, coloca um dos principais desafios do nosso século que será satisfazer a maior procura de cuidados de saúde, adaptá-los a esta nova realidade e mantê-los económica e socialmente viáveis numa sociedade reflexamente com menos população ativa. ${ }^{1-4}$

O envelhecimento da população é um fenómeno global. Prevê-se que os $11 \%$ de população com idade su- perior a 60 anos, existentes em 2006, aumentem para $22 \%$ em $2050 .^{5}$

\section{Doença crónica e polimedicação}

A população idosa apresenta com mais frequência pluripatologia e, por isso, é a que tendencialmente utiliza mais os serviços de saúde, sendo também a maior consumidora de fármacos. A polimedicação (PM) tem

1. Centro Hospitalar Trás-os-Montes e Alto Douro. Escola Superior de Enfermagem Dr. José Timóteo Montalvão Machado.

2. Médica de medicina geral e familiar. USF Manuel Rocha Peixoto - Braga. 
sido definida de várias formas, nomeadamente utilização de vários medicamentos, a toma de mais medicamentos do que os indicados, o regime terapêutico que inclua pelo menos um medicamento desnecessário e/ou o uso de medicamentos que podem causar reações adversas/interações medicamentosas. ${ }^{6-8}$

A PM pode ser classificada em polimedicação minor (PMMinor), de dois a quatro fármacos, e polimedicação major (PMMajor) quando se utilizam cinco ou mais fármacos, ${ }^{9-10}$ sendo a cronicidade definida como aquela que é realizada por um período nunca inferior a três meses. ${ }^{11}$

Num estudo efetuado nos Centros de Saúde de Queluz e do Lumiar constatou-se que, dos 571 utentes com uma média de idade de 58,2 anos, o consumo médio de medicamentos era de 2,2 .

Este número aumentou para 3,9 nos utentes com mais de 65 anos. Observou-se ainda que a PMMinorna população idosa era de $39,6 \%$ e a PMMajor era de $37,1 \% .{ }^{10}$ Num estudo efetuado em 3.481 utentes da zona Norte de Portugal (Porto, Braga, Viana do Castelo, Vila Real e Bragança), tendo por base a lista de utentes de 45 médicos de MGF, observou-se que, no que diz respeito à medicação crónica, o número médio de fármacos em maiores de 18 anos era de 2,94 e que $91,1 \%$ dos utentes com idade igual ou superior a 75 anos faziam medicação crónica. ${ }^{12}$

Nos idosos, a PM deve-se essencialmente ao número de doenças crónicas associadas à idade, ${ }^{11}$ a falhas na revisão ou descontinuação da medicação e à dificuldade económica em obter terapêuticas não farmacológicas. $^{13-14}$

\section{Polimedicação e reações adversas}

Na medida em que o número de medicamentos prescritos aumenta é também de esperar um aumento das reações adversas e interações medicamentosas. ${ }^{6-8}$

Evento adverso pode ser definido como uma reação, esperada ou não, num doente, devido à toma de um fármaco prescrito, ou não, pelo médico. ${ }^{15-16}$

A reação adversa ao medicamento (RAM) é definida, pela OMS, como qualquer efeito que seja prejudicial ou indesejado que se apresente após a administração dos medicamentos em doses normalmente utilizadas no Homem para profilaxia, diagnóstico ou tratamento de doença ou para modificação de funções fisiológicas. ${ }^{17}$
A PIM é definida como o uso sem evidência (medicamento sem indicação terapêutica válida), uso de um medicamento errado (dose ou esquema posológico desajustado, inadequado ou numa duração desadequada) ou subutilização (não utilização de um medicamento potencialmente benéfico). ${ }^{18-20}$

Em 1989, Beers e colaboradores consideraram que o uso de medicamentos podia constituir um auxílio precioso, mas podia também ser responsável por doença iatrogénica, principalmente no doente geriátrico. ${ }^{21}$ Assim, em 1991, um consenso de peritos elaborou uma lista de medicamentos potencialmente inapropriados de forma a auxiliar a deteção de possíveis riscos de iatrogenia medicamentosa, que ficou conhecida como Critérios de Beers. ${ }^{22-23}$ Os Critérios de Beers predominaram na literatura internacional desde a sua descrição em 1991, tendo sido posteriormente ajustados e atualizados, tendo em conta os novos conhecimentos científicos. ${ }^{22-23}$

Em 2008, os Critérios de Beers foram operacionalizados para Portugal tendo em conta a última atualização desta lista de medicamentos potencialmente inapropriados nos idosos. $^{24}$

Já em 2015, a Sociedade Americana de Geriatria volta a atualizar as listas de PIMs. Nestas novas listas, para cada fármaco avaliado existe uma fundamentação teórica sobre motivos pelos quais não devem ser usados em idosos, assim como recomendações clínicas, nível de evidência científica e grau de recomendação. ${ }^{25}$ Mais recentemente surgiram os critérios STOPP/START aplicáveis aos cuidados de saúde primários, que incluem medicamentos potencialmente omissos.

Num estudo francês com 150 pacientes com 75 anos ou mais, admitidos durante dois meses no hospital de Charpennes, identificaram-se 40 pacientes $(26,7 \%)$ idosos com prescrições de PIMs, sendo que a classe farmacológica mais comummente detetada foi a dos psicotrópicos. $^{26}$

Este estudo pretende identificar um dos problemas associado ao envelhecimento das populações: aumento do número de fármacos e do risco de provocar eventuais interações medicamentosas. A análise à PM e PIMs e a fatores contributivos pretende ajudar a planear futuras intervenções educacionais dos médicos prescritores com o objetivo de ajudar a melhorar os cuidados assistenciais ao paciente idoso. 
Os objetivos do presente estudo foram: conhecer a terapêutica crónica nos idosos com idade igual ou superior a 75 anos numa unidade de saúde familiar de Braga, determinar a frequência (relativa e absoluta) de polimedicação e medicação potencialmente inapropriada e identificar fatores associados.

\section{MATERIAL E MÉTODOS}

Estudo observacional, transversal, com vertentes descritiva e analítica, do tipo exploratório. Realizado na Unidade de Saúde Familiar Manuel Rocha Peixoto (USF-MRP), Agrupamento do Centro de Saúde Cávado I - Braga, tendo por base a lista de utentes de dois médicos da unidade.

A população alvo foi constituída por utentes, de ambos os sexos, com idade igual ou superior a 75 anos, inscritos nos ficheiros clínicos desses dois médicos. O número total de indivíduos elegíveis para este estudo foi de 198.

A amostra investigada foi do tipo aleatória, simples e sem reposição.

A dimensão da amostra foi calculada através do programa Open Epi, com base numa prevalência de 50\%, visto que se desconhece, na população em estudo, a prevalência do principal objetivo.

Obteve-se, assim, uma amostra de 198 utentes que foi posteriormente aleatorizada através do programa informático Random.org, usando os primeiros 131 números.

\section{Critérios de inclusão}

Utentes da USF-MRP, com idade igual ou superior a 75 anos de idade, inscritos na lista de utentes de dois médicos da USF-MRP.

\section{Critérios de exclusão}

Utentes incapazes de compreender ou responder ao questionário aplicado; que se encontrassem hospitalizados; que residissem ou estivessem institucionalizados fora do concelho de Braga; cujo contacto telefónico não tivesse sido possível, por não existir no processo, por este já não ser o seu contacto pessoal ou por não atender a chamada (foram efetuados sempre, pelo menos, duas tentativas de contacto em dias e horas diferentes); que não se encontrassem no domicílio à data de marcação da visita domiciliária.
Método e recolha de dados

O guião de entrevista foi construído pelos investigadores, após constatar a inexistência de formulário que contemplasse os objetivos propostos. Foi efetuado um estudo piloto a fim de testar e averiguar a eficácia do método e a aplicabilidade do formulário de investigação.

O guião de entrevista é constituído por duas secções. A primeira é a secção «Caracterização do Utente» da qual fazem parte questões respeitantes à caracterização socio-demográfica, clínica e de autoperceção da saúde. A segunda é a «Secção Medicação Crónica», que inclui questões de escolha múltipla e espaços para preenchimento sobre o esquema terapêutico.

Os utentes foram contactados telefonicamente para marcação da entrevista, tendo esta sido realizada no domicílio.

Para a recolha de dados utilizou-se o guião de entrevista após o utente ter assinado o consentimento informado. Apenas foi considerada a informação oral fornecida pelo utente e a visualização, com devido registo, da terapêutica efetuada por este (foi pedido, a cada utente, que, no momento da entrevista, tivesse disponível toda a medicação efetuada). O método de colheita foi totalmente anónimo, sendo as questões colocadas de forma a não identificar o utente.

Os utentes foram previamente informados do âmbito da investigação e teve-se em consideração a Lei n. ${ }^{\circ}$ 12/2005, de 26 de janeiro - Informação genética pessoal e informação de saúde, nomeadamente o artigo $4 .^{\circ}$ (tratamento da informação de saúde).

Toda a informação obtida foi introduzida numa base de dados no programa informático Statistical Package for the Social Sciences vs. 20.0 (SPSS 20.0) e protegida com palavra-chave.

A avaliação da utilização dos medicamentos foi retrospetiva e não interventiva.

O estudo foi submetido à Comissão de Ética para a Saúde, da Administração Regional de Saúde do Norte, tendo sido aprovado por parte desta entidade e pela Coordenadora da USF-MRP.

Os dados constantes no guião de entrevista foram introduzidos numa base de dados no programa informático SPSS 20.0, seguindo-se a sua depuração para corrigir eventuais erros de registo.

$\mathrm{Na}$ análise univariada as variáveis categóricas foram 
descritas através de frequências absolutas e relativas. As variáveis contínuas foram descritas através da média e desvio-padrão. A definição do intervalo de confiança de $95 \%(p=0,05)$ foi efetuada para rejeitar as hipóteses nulas. Para a análise bivariada, análise da relação entre duas variáveis qualitativas (nominais), utilizou-se a prova Qui quadrado $(\chi 2)$.

Para averiguar as PIM foram usados os Critérios de Beers.

\section{RESULTADOS}

Dos 131 utentes selecionados, aleatoriamente 86 participaram no estudo, perfazendo uma taxa de participação de 65,6\%. Dos 45 utentes que não participaram, 35 (77,8\%) preenchiam os critérios de exclusão e 10 recusaram participar.

\section{Caracterização socio-demográfica}

Os participantes tinham entre 78 e 100 anos, com média de 84 anos. A distribuição por sexo revela uma predominância do sexo feminino, 63 (73,3\%).

Verificou-se uma percentagem de analfabetismo de $36,0 \%$, sendo a mais frequente, seguindo-se o $1^{\circ}$ ciclo incompleto $(32,6 \%)$ e o $1^{\circ}$ ciclo completo $(19,8 \%)$, perfazendo $89 \%$ da amostra.

Na maioria eram viúvos $(54,7 \%)$. De relevar que $69,8 \%$ da amostra viviam em freguesias que integram a cidade e que mais de metade da amostra (57\%) tinha rendimento individual até um salário mínimo. Outras características relevantes encontram-se no Quadro I.

\section{Caracterização dos cuidados de saúde e principais doenças associadas}

Relativamente ao número de consultas nos últimos 12 meses verificou-se que o número mínimo (zero consultas) ocorreu em quatro utentes e o número máximo ocorreu em um utente (20 consultas). A média de consultas por ano é de 5,1, o que significa aproximadamente uma consulta a cada dois meses. Em relação ao número de internamentos, 63 utentes $(73,3 \%)$ não estiveram internados nos últimos 12 meses. As doenças identificadas com maior prevalência foram HTA, dislipidemia e doenças osteoarticulares, presentes em 55 (74,3\%), $41(55,4 \%)$ e $47(63,5 \%)$ utentes, respetivamente.

\begin{tabular}{|c|c|}
\hline \multicolumn{2}{|c|}{$\begin{array}{l}\text { QUADRO I. Caracterização social e demográfica dos } \\
\text { utentes }(n=86)\end{array}$} \\
\hline Variáveis & $\mathbf{N}(\%)$ \\
\hline Idade* (média) & $84,1(4,8)$ \\
\hline \multicolumn{2}{|l|}{ Sexo } \\
\hline Feminino & $63(73,3)$ \\
\hline Masculino & $23(26,7)$ \\
\hline \multicolumn{2}{|l|}{ Escolaridade } \\
\hline Sem escolaridade & $31(36,0)$ \\
\hline $1^{\circ}$ Ciclo incompleto & $28(32,6)$ \\
\hline $1^{\circ}$ Ciclo & $17(19,8)$ \\
\hline $2^{\circ}$ Ciclo & $0(0)$ \\
\hline $3^{\circ}$ Ciclo & $3(3,5)$ \\
\hline Secundário & $4(4,7)$ \\
\hline Licenciatura & $3(3,5)$ \\
\hline \multicolumn{2}{|l|}{ Estado civil } \\
\hline Casado & $24(27,9)$ \\
\hline Vive maritalmente & $1(1,2)$ \\
\hline Viúvo & $47(54,7)$ \\
\hline Solteiro & $13(15,1)$ \\
\hline Separado & $1(1,2)$ \\
\hline \multicolumn{2}{|l|}{ Tipo de Freguesia } \\
\hline Que integra a cidade & $60(69,8)$ \\
\hline Predominantemente urbana & $10(11,6)$ \\
\hline Mediamente urbana & $16(18,6)$ \\
\hline \multicolumn{2}{|l|}{ Com quem reside } \\
\hline Sozinho & $13(15,1)$ \\
\hline Cônjuge & $18(20,9)$ \\
\hline Cônjuge e filho(s)/com filho(s) & $30(34,9)$ \\
\hline Com outros familiares & $3(3,5)$ \\
\hline Institucionalizado & $22(26)$ \\
\hline \multicolumn{2}{|l|}{ Rendimento individual } \\
\hline Até 1 salário mínimo & $49(57,0)$ \\
\hline De 1 a 2 salários mínimos & $18(20,9)$ \\
\hline Mais de 2 salários mínimos & $6(7,0)$ \\
\hline Não sabe / Não responde & $13(15)$ \\
\hline \multicolumn{2}{|l|}{ Atividade física } \\
\hline Não pratica exercício & $51(59,3)$ \\
\hline $1-2$ vezes por semana & $16(18,6)$ \\
\hline 3 ou mais vezes por semana & $19(22,1)$ \\
\hline
\end{tabular}

* As distribuições correspondem a média (desvio-padrão) 


\begin{tabular}{|c|c|c|}
\hline \multirow{2}{*}{$\begin{array}{l}\text { Grupos farmacoterapêuticos } \\
\text { Grupo } 2 \text { - Sistema Nervoso Central (SNC) } \\
\qquad(n=118 ; 22 \%)\end{array}$} & \multicolumn{2}{|l|}{ Classes terapêuticas ( $n$; \%) } \\
\hline & 2.9. Psicofármacos & $81 ; 15,1 \%$ \\
\hline $\begin{array}{l}\text { Grupo } 3 \text { - Aparelho Cardiovascular } \\
\qquad(n=213 ; 39,7 \%)\end{array}$ & $\begin{array}{l}\text { 3.4. Anti-hipertensores } \\
\text { 3.5. Vasodilatadores } \\
\text { 3.7. Antidislipidémicos }\end{array}$ & $\begin{array}{r}122 ; 22,7 \% \\
23 ; 4,3 \% \\
47 ; 8,8 \%\end{array}$ \\
\hline $\begin{array}{l}\text { Grupo } 4 \text { - Sangue } \\
\qquad(n=59 ; 11 \%)\end{array}$ & 4.3. Anticoagulantes e antitrombóticos & $54 ; 10 \%$ \\
\hline $\begin{array}{l}\text { Grupo 5 - Aparelho Respiratório } \\
\qquad(n=24 ; 4,5 \%)\end{array}$ & 5.1 Antiasmáticos e broncodilatadores & $23 ; 4,3 \%$ \\
\hline $\begin{array}{r}\text { Grupo 6 - Aparelho Digestivo } \\
(n=28 ; 5,2 \%)\end{array}$ & 6.2 Antiácidos e antiulcerosos & $19 ; 3,5 \%$ \\
\hline $\begin{array}{l}\text { Grupo } 7 \text { - Aparelho Geniturinário } \\
\qquad(n=19 ; 3,5 \%)\end{array}$ & 7.4.2. Usados na perturbação da micção & $18 ; 3,3 \%$ \\
\hline $\begin{array}{l}\text { Grupo } 8 \text { - Hormonas e medicamentos usados } \\
\text { no tratamento de doenças endócrinas } \\
\qquad(n=25 ; 4,6 \%)\end{array}$ & $\begin{array}{l}\text { 8.3 Hormonas da tiroide e antitiroideus } \\
8.4 \text { Insulinas, ADO e glucagom }\end{array}$ & $\begin{array}{r}6 ; 1,1 \% \\
17 ; 3,2 \%\end{array}$ \\
\hline $\begin{array}{l}\text { Grupo } 9 \text { - Aparelho Locomotor } \\
\qquad(n=34 ; 6,3 \%)\end{array}$ & $\begin{array}{l}\text { 9.1. Anti-inflamatórios não esteróides } \\
\text { 9.3. Tratamento da gota } \\
\text { 9.4. Tratamento da osteoporose } \\
\text { 9.5. Atuam no osso e seu metabolismo }\end{array}$ & $\begin{array}{r}12 ; 2,2 \% \\
7 ; 1,3 \% \\
5 ; 0,9 \% \\
10 ; 1,8 \%\end{array}$ \\
\hline Total & & $520 ; 100 \%$ \\
\hline
\end{tabular}

midores crónicos de pelo menos um fármaco. A média de fármacos por utente foi de 6,2. O número máximo de fármacos num utente foi de 14 .

Dos 83 utentes medicados, 20 $(24,1 \%)$ consumiam de dois a quatro medicamentos (PMMinor) e $62(74,7 \%)$ consumiam cinco ou mais (PMMajor), de forma crónica.

Tendo em conta os parâmetros anteriores e comparando-os com o sexo verificou-se que dos 63 utentes do sexo feminino, $23,8 \%$ registaram

Perceção sobre saúde e risco para múltipla medicação

Globalmente, 57\% dos utentes tinha uma autoperceção negativa da sua saúde e apenas metade dos utentes preparava a sua própria medicação $(51,2 \%)$. Da amostra, 40,7\% desconhecia a finalidade da medicação que consumia. Dos 86 utentes, 27 (31,4\%) desconhecia o conceito de «interação medicamentosa» e riscos associados.

\section{Análise da medicação crónica}

\section{Médico prescritor}

O grupo de utentes estava sob prescrição/orientação de vários médicos em simultâneo $(65,1 \%$ médico de medicina geral e familiar, $44,2 \%$ médico privado e $34,9 \%$ médico hospitalar), sendo que 48 utentes $(55,8 \%)$ tinham apenas um médico prescritor.

\section{Número e tipo de medicação crónica (PMMinor e} PMMajor): análise por género e escalão etário

Dos 86 utentes estudados, 83 (96,5\%) eram consu-
PMMinor e 69,8\% PMMajor. Nos 23 utentes do sexo masculino registaram-se 21,7\% de PMMinor e 78,3\% com PMMajor, não existindo neste grupo qualquer utente sem PM e verificando-se uma tendência para maior PMMajor nos idosos do sexo masculino.

Em relação ao escalão etário verificou-se que nos escalões etários 75-84 anos (51 utentes) e em $\geq 85$ anos (35 utentes) se verificaram respetivamente $29,4 \%$ e $66,7 \%$ de idosos com PMMinor e 14,3\% e 80,0\% com PMMajor, verificando-se tendência crescente de PMMajor com o avançar da idade.

\section{Análise dos fármacos prescritos}

Dos 536 fármacos prescritos, as classes terapêuticas mais consumidas, segundo o Prontuário Terapêutico, foram os fármacos para o sistema cardiovascular (213; $39,7 \%$ ) e sistema nervoso central (SNC) $(118 ; 22 \%)$ (Quadro II).

Os 16 (3\%) fármacos não representados no Quadro II estão distribuídos pelos grupos 1, 10, 11 e 15 do Pron- 
QUADRO III. Número de PIM nos utentes da amostra com PIM

\begin{tabular}{|c|c|c|c|c|c|}
\hline \multicolumn{3}{|c|}{$\begin{array}{l}\text { Critérios de Beers - } \\
\text { Operacionalização Portugal } \\
\text { (CB-OP) (2008) }\end{array}$} & \multicolumn{3}{|c|}{$\begin{array}{l}\text { Atualização Critérios de Beers } \\
\text { pela American Geriatrics } \\
\text { Society (AGS) (2012) }\end{array}$} \\
\hline $\begin{array}{l}\text { Número de PIM } \\
\text { por utente }\end{array}$ & $n$ & $\%$ & $\begin{array}{c}\text { Número de PIM } \\
\text { por utente }\end{array}$ & $n$ & $\%$ \\
\hline 1 & 22 & 25,6 & 1 & 31 & 36,0 \\
\hline 2 & 9 & 10,5 & 2 & 8 & 9,3 \\
\hline \multirow[t]{2}{*}{3} & 1 & 1,2 & 3 & 2 & 2,3 \\
\hline & & & 4 & 1 & 1,2 \\
\hline Total & 32 & 37,2 & Total & 42 & 48,8 \\
\hline
\end{tabular}

tuário Terapêutico, que correspondem respetivamente a medicamentos anti-infeciosos, medicação antialérgica, nutrição e medicamentos usados nas afeções oculares.

Os quatro medicamentos com maior prevalência neste estudo foram: ácido acetilsalicílico, sinvastatina, furosemida e lorazepam prescritos respetivamente em $34,31,26$ e 17 utentes.

\section{Análise dos fármacos prescritos - Medicação potencialmente inapropriada (PIM) - Critérios de Beers}

Trinta e dois utentes $(37,2 \%)$ em estudo tinham na sua prescrição crónica um PIM segundo a operacionalização para Portugal dos Critérios de Beers (CB-OP) (2008) e este número sobe para 42 utentes (48,8\%), praticamente um utente em cada dois, quando se aplica a atualização destes critérios efetuada pela American Geriatrics Society (AGS) em 2012.

Dos utentes medicados com PIM, alguns faziam mais que um medicamento potencialmente inapropriado (Quadro III).

Em relação ao escalão etário dos 75-84 anos, observaram-se $41,2 \%$ de utentes a fazer PIM segundo CP-OP e $52,9 \%$ PIM segundo AGS. No escalão $\geq 85$ anos verificouse $31,4 \%$ PIM segundo CP-OP e 57,1\% PIM segundo AGS.

Os CB-OP identificam os fármacos considerados PIM e classifica-os segundo o grau de inadequação das diferentes substâncias em «Ligeiro» ou «Elevado», de acordo com o seu potencial em desenvolver RAMs. Dos 43 PIM identificados (em 32 utentes), 39 (90,7\%) foram classificados com o grau de inadequação «Elevado».
Dos fármacos considerados pelos CB como PIM, os que foram mais vezes prescritos são os que atuam no $\mathrm{SNC}$, destacando-se as benzodiazepinas (BDZ), principalmente as de curta duração de ação (12 utentes). Os PIM que atuam no SNC, segunda a AGS (2012), são 43, correspondendo a $75,4 \%$ do total de fármacos potencialmente inapropriados.

Fatores (socio-demográficos, cuidados de saúde, perceção e risco da múltipla medicação) que se relacionam com a maior incidência de PMMajor e PIM

Após análise estatística não se encontrou significado estatístico em relação às variáveis independentes (faixa etária, sexo, escolaridade, tipo de freguesia e rendimento individual), quando comparadas com as variáveis dependentes, PMMajor e NoPIM-CB-OP.

\section{Variável dependente PMMajor}

A variável dependente PMMajor apresentou significado estatístico com as variáveis independentes autoperceção da saúde, preparação da medicação, conhecimento da finalidade da medicação e número de prescritores (Quadro IV).

\section{Variável dependente PIM CB-OP}

A variável dependente PIM CB-OP apresentou significado estatístico com as variáveis independentes autoperceção da saúde, preparação da medicação e conhecimento da finalidade da medicação (Quadro V).

\section{DISCUSSÃO E CONCLUSÃO}

\section{Contexto do estudo e amostra estudada}

A amostra selecionada e investigada neste estudo foi aleatória, simples e sem reposição de utentes inscritos numa USF de Braga. Foram considerados válidos para análise 86 utentes, com as características socio-demográficas apresentadas anteriormente (Quadro I). Comparativamente a outros estudos ${ }^{24,34} \mathrm{e}$, como definido previamente, a análise incide sobre o grupo etário acima dos 75 anos.

$\mathrm{O}$ facto de se ter feito entrevista pessoalmente, tendo-se pedido ao utente que se fizesse acompanhar de toda a medicação efetuada, permitiu diminuir o viés de memória em relação à medicação. Este viés está presente noutros trabalhos consultados, que se basearam na consulta do processo clínico e na medicação pres- 


\begin{tabular}{|c|c|c|c|c|}
\hline Variáveis & $\begin{array}{c}\text { Sem PMMajor } \\
n=24\end{array}$ & $\begin{array}{l}\text { Com PMMajor } \\
\qquad \begin{array}{l}N=62\end{array}\end{array}$ & $\begin{array}{c}\text { Teste } \\
\text { estatístico }\end{array}$ & $p$ \\
\hline $\begin{array}{l}\text { Autoperceção da saúde* } \\
\text { Negativa } \\
\text { Positiva } \\
\text { Não sabe/Não responde** }\end{array}$ & $\begin{array}{r}9(37,5) \\
23(54,2) \\
2(8,3)\end{array}$ & $\begin{array}{r}40(64,5) \\
17(27,4) \\
5(8,1)\end{array}$ & $\chi 2(1)=5,772$ & 0,016 \\
\hline $\begin{array}{l}\text { No internamentos nos últimos } \\
12 \text { meses* } \\
0 \text { Internamentos } \\
1 \text { Internamento } \\
\text { Não sabe/Não responde** }\end{array}$ & $\begin{array}{r}20(83,3) \\
4(16,7) \\
0(0,0)\end{array}$ & $\begin{array}{r}43(69,4) \\
18(29,0) \\
1(1,6)\end{array}$ & $\chi 2(1)=1,481$ & 0,224 \\
\hline 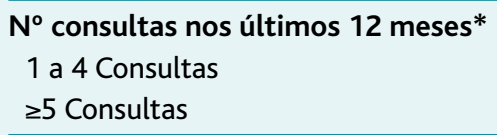 & $\begin{array}{r}17(70,8) \\
5(20,8)\end{array}$ & $\begin{array}{l}29(46,8) \\
25(40,3)\end{array}$ & & 0,057 \\
\hline $\begin{array}{l}\text { Preparação da medicação* } \\
\text { Próprio } \\
\text { Familiar } \\
\text { Outro cuidador }\end{array}$ & $\begin{array}{r}18(75,0) \\
3(12,5) \\
3(12,5)\end{array}$ & $\begin{array}{l}26(41,9) \\
19(30,6) \\
17(27,4)\end{array}$ & $\chi 2(2)=7,580$ & 0,023 \\
\hline $\begin{array}{l}\text { Conhecimento da finalidade da } \\
\text { medicação* } \\
\text { Sim } \\
\text { Não }\end{array}$ & $\begin{array}{r}22(91,7) \\
2(8,3)\end{array}$ & $\begin{array}{r}29(46,8 \%) \\
33(53,2)\end{array}$ & $\chi 2(1)=14,448$ & $<0,001$ \\
\hline $\begin{array}{l}\text { Conhecimento de interações } \\
\text { medicamentosas* } \\
\text { Sim } \\
\text { Não }\end{array}$ & $\begin{array}{r}9(37,5) \\
15(62,5)\end{array}$ & $\begin{array}{l}18(29,0) \\
44(71,0)\end{array}$ & $\chi 2(1)=0,576$ & 0,448 \\
\hline $\begin{array}{l}\text { Conhecimento de riscos associados } \\
\text { às interações medicamentosas* } \\
\text { Sim } \\
\text { Não }\end{array}$ & $\begin{array}{r}9(37,5) \\
15(62,5)\end{array}$ & $\begin{array}{l}18(29,0) \\
44(71,0)\end{array}$ & $\chi 2(1)=0,576$ & 0,448 \\
\hline $\begin{array}{l}N^{\circ} \text { de prescritores** } \\
1 \text { Prescritor } \\
\text { Mais de } 1 \text { prescritor }\end{array}$ & $\begin{array}{r}16(66,7) \\
5(20,8)\end{array}$ & $\begin{array}{l}32(51,6) \\
30(48,4)\end{array}$ & & 0,049 \\
\hline
\end{tabular}

*As distribuições correspondem a $n$ (\%)

crita, que eventualmente poderia não estar a ser feita pelo utente, não sendo, assim, responsável por interações medicamentosas. Tentou-se também que a amostra estudada se aproximasse o mais possível da população geral. Neste estudo, o facto de se ter aleatorizado todos os utentes e não apenas os utilizadores dos serviços, tende a representar toda a população servida pela respetiva USF e não apenas os utilizadores dos cuida- dos de saúde primários (CSP), conforme se verifica noutros estudos. ${ }^{12,28-30}$

$\mathrm{O}$ instrumento de medição foi um guião de entrevista construído de forma a eliminar a probabilidade de questionários inválidos e de má interpretação, assim como colmatar a dificuldade que poderia advir da baixa escolaridade da amostra $(36,0 \%$ de analfabetismo).

A amostra em estudo tinha uma média de idade de 84 anos, com predominância do sexo feminino (73,3\%), idêntica à encontrada noutros estudos portugueses $\quad 72,8 \%^{30}$ e internacionais $74,4 \% .{ }^{27,36-37}$

Neste estudo, mais de metade dos utentes (57\%) autopercecionam a sua saúde como negativa, valor mais elevado do que o encontrado no estudo efetuado nos Centros de Saúde do Lumiar e de Queluz ${ }^{10}$ e na população portuguesa. ${ }^{31}$ Poder-se-á pensar em possíveis explicações considerando o espaço geográfico-económico em que se insere (Minho versus Área Metropolitana de Lisboa) e/ou os elevados valores nos índices relacionados com o elevado número de fármacos por utente registado nesta população, o que poderá indiciar também elevados índices de patologia crónica. 
Verifica-se que 25,6\% dos utentes tiveram um internamento nos últimos doze meses e que o número médio de consultas anuais é de cinco, revelando maior fragilidade e necessidade de acompanhamento médico neste grupo etário.

Este grande número de consultas anuais/internamentos releva a necessidade de intervenção médica e reafirma a necessidade de alerta para o risco de doença iatrogénica medicamentosa, reforçando, e sendo possível justificação para, a análise subsequente realizada neste estudo.

Apesar de a amostra aleatória ser retirada da lista de utentes de uma USF, apenas 56 utentes tinham o seu médico de família como um dos prescritores, existindo nesta amostra um apreciável número de utentes a ser seguido fora dos CSP.

\section{Polimedicação, respetivos}

determinantes

$\mathrm{Na}$ amostra estudada encontraram-se $96,5 \%$ de utentes consumidores de pelo menos um medicamento, de forma crónica, valor muito próximo dos 95,1\% encontrados num estudo efetuado em oito países europeus e igual ao encontrado no Reino Unido, onde se estudou a medicação efetuada nos sete dias precedentes à colheita da informação. ${ }^{27}$ Está também muito próximo dos 96,9\% encontrados em utentes maiores de 70 anos num centro de saúde do Porto, ${ }^{28}$ sendo no entanto mais elevada do que a percentagem encontrada num estudo de prevalência no Norte de Portugal, que foi de $91,1 \%$ em utentes com mais de 75 anos. ${ }^{12}$

Desconhece-se qual a prevalência de PM nos idosos portugueses mas, em estudos efetuados em Portugal, o

\begin{tabular}{|c|c|c|c|c|}
\hline Variáveis & $\begin{array}{c}\text { Sem PIM } \\
n=54\end{array}$ & $\begin{array}{c}\text { Com PIM } \\
N=32\end{array}$ & $\begin{array}{c}\text { Teste } \\
\text { estatístico }\end{array}$ & $\mathbf{P}$ \\
\hline $\begin{array}{l}\text { Autoperceção da saúde* } \\
\text { Negativa } \\
\text { Positiva }\end{array}$ & $\begin{array}{l}28(51,9) \\
24(44,4)\end{array}$ & $\begin{array}{r}21(65,6) \\
6(18,8)\end{array}$ & & 0,038 \\
\hline $\begin{array}{l}N^{\circ} \text { internamentos nos últimos } \\
12 \text { meses* } \\
0 \text { Internamentos } \\
1 \text { Internamento }\end{array}$ & $\begin{array}{l}39(72,2) \\
14(25,9)\end{array}$ & $\begin{array}{r}24(75,0) \\
8(25,0)\end{array}$ & & 0,885 \\
\hline $\begin{array}{l}N^{\circ} \text { consultas nos últimos } 12 \text { meses* } \\
1 \text { a } 4 \text { Consultas } \\
\geq 5 \text { Consultas }\end{array}$ & $\begin{array}{l}28(51,9) \\
18(33,3)\end{array}$ & $\begin{array}{l}18(56,2) \\
12(37,5)\end{array}$ & & 0,564 \\
\hline $\begin{array}{l}\text { Preparação da medicação* } \\
\text { Próprio } \\
\text { Familiar } \\
\text { Outro cuidador }\end{array}$ & $\begin{array}{l}34(63,0) \\
10(18,5) \\
10(18,5)\end{array}$ & $\begin{array}{l}10(31,2) \\
12(37,5) \\
10(31,2)\end{array}$ & $\chi 2(2)=8,180$ & 0,017 \\
\hline $\begin{array}{l}\text { Conhecimento da finalidade da } \\
\text { medicação* } \\
\text { Sim } \\
\text { Não }\end{array}$ & $\begin{array}{l}38(70,4) \\
16(29,6)\end{array}$ & $\begin{array}{l}13(40,6) \\
19(59,4)\end{array}$ & $\chi 2(1)=7,366$ & 0,007 \\
\hline $\begin{array}{l}\text { Conhecimento de interações } \\
\text { medicamentosas* } \\
\text { Sim } \\
\text { Não }\end{array}$ & $\begin{array}{l}21(38,9) \\
33(61,1)\end{array}$ & $\begin{array}{r}6(18,8) \\
26(81,2)\end{array}$ & $\chi 2(1)=3,784$ & 0,052 \\
\hline $\begin{array}{l}\text { Conhecimento de riscos associados } \\
\text { às interações medicamentosas* } \\
\text { Sim } \\
\text { Não }\end{array}$ & $\begin{array}{l}21(38,9) \\
33(61,1)\end{array}$ & $\begin{array}{r}6(18,8) \\
26(81,2)\end{array}$ & $\chi 2(1)=3,784$ & 0,052 \\
\hline $\begin{array}{l}\text { No de prescritores }^{* *} \\
1 \text { Prescritor } \\
\text { Mais de } 1 \text { prescritor }\end{array}$ & $\begin{array}{l}32(59,3) \\
19(35,2)\end{array}$ & $\begin{array}{l}16(50,0) \\
16(50,0)\end{array}$ & & 0,252 \\
\hline
\end{tabular}

* As distribuições correspondem a $n$ (\%) consumo de três ou mais fármacos teve prevalências entre $56 \%{ }^{29} \mathrm{e} 65 \% .^{10,28}$ Neste último estudo, a média de fármacos por utente idoso foi de 3,9, a PMMinor de $39,6 \%$ e a PMMajor de $37,1 \%$. A amostra estudada, no presente estudo, tinha uma média de 6,2 fármacos por utente, 23,3\% de PMMinor e 72,1\% de PMMajor, valores díspares do estudo efetuado nos Centros de Saúde de Queluz e do Lumiar e dos $51 \%$ encontrados no estudo europeu referido anteriormente, mas próximo dos valores encontrados na Finlândia - 73,3\%. ${ }^{27}$ 
Os 72,1\% de PMMajor afastaram-se dos valores encontrados noutros estudos efetuados em Portugal e na Europa. Isto pode ser explicado pelo facto de, apesar de nos estudos referidos a amostra também ser aleatória, incluiu idosos com idade igual ou superior a 65 anos, enquanto no presente estudo a amostra inclui idosos com idade igual ou superior a 75 anos, provavelmente com maior morbilidade e medicação.

A PMMajor foi mais prevalente no sexo masculino ao contrário dos três estudos realizados em Portugal. ${ }^{10,28-29}$ Isto poderá, em parte, ser explicado pelo facto de nesses estudos ser considerado o uso de anticoncecionais orais, já que incluem toda a população, incluindo jovem/adulta feminina.

Tendo em conta a idade, verificou-se um aumento progressivo do consumo de medicamentos, sendo mais prevalente no escalão etário de idosos com idade igual ou superior a 85 anos. Este resultado era esperado pois, associada muitas vezes à idade, existe também uma maior comorbilidade, o que também se verificou nos estudos realizados anteriormente..$^{10,27-29}$

A classe terapêutica mais utilizada foi a do sistema cardiovascular seguindo-se a do SNC, como também observado noutros estudos. ${ }^{12-13,28,30,32}$

\section{Medicação potencialmente inapropriada}

A prescrição de PIM em idosos na amostra estudada foi de $37,2 \%$ segundo os CB-OP e de $48,8 \%$ segundo a atualização da AGS. Em diferentes estudos, a percentagem de PIM em ambulatório varia de $13,4 \%$ a $31 \%$ nos EUA, ${ }^{32-33} 18,9 \%$ na Noruega ${ }^{34}$ e $19,8 \%$ num estudo que envolveu oito países europeus, ${ }^{27}$ não atingindo em nenhum dos casos uma percentagem tão elevada como a encontrada no presente estudo. Percentagem semelhante à encontrada, segundo os CB-OP, foi observada num estudo efetuado em doze farmácias portuguesas, onde se observaram $27,7 \%$ de PIM segundo os CB (1997) e de $38,5 \%$ segundo os CB, versão 2003, onde seria expectável encontrar valores mais altos, uma vez que se tratava de uma população polimedicada com dois ou mais fármacos que recorriam às farmácias. ${ }^{35} \mathrm{Os}$ valores mais elevados de PIM, encontrados na revisão bibliográfica, dizem respeito à República Checa $(41,1 \%),{ }^{27}$ Índia $(41,2 \%)^{36}$ e Japão $(43,6 \%) .{ }^{37}$

Dos PIM, 90,7\% eram classificados com grau de inadequação «Elevado», valor superior ao encontrado no estudo realizado em farmácias portuguesas $(87,7 \%) .^{35}$ Os PIM segundo CB-OP mais vezes prescritos são os que atuam no SNC, destacando-se as BDZ que correspondem a 44\%. O diazepam, BDZ de longa duração, é o fármaco PIM mais prescrito, facto que também ocorre noutros estudos, nomeadamente no português ${ }^{35} \mathrm{e}$ nos estudos europeus já referidos. ${ }^{24,27,34}$

As BDZ correspondem a $44 \%$ de todos os PIM segundo os CO-OP e a 75,4\% segundo a atualização da AGS. As de curta ação, no seu conjunto, são os fármacos mais frequentes (27,9\% CB-OP; 45,6\% AGS) sendo, como tal, de grande relevância quando se sabe que as BDZ estão associadas ao maior risco de fraturas, tão importante nesta faixa etária. ${ }^{38-40}$ Segundo este último estudo, o uso de BDZ leva a um aumento potencial do risco de fratura da anca, que varia entre $1,8 \%$ e $8,2 \%$ nos cinco países europeus e Estados Unidos. Estes fármacos devem ser prescritos de forma criteriosa pois, para além das fraturas, estão ainda associados, principalmente nesta população, ao aumento de RAMs como sonolência, confusão e quedas. ${ }^{33}$

\section{Fatores de correlação}

Na literatura observa-se que a frequência de PIM aumenta com a idade ${ }^{27,32,41-42} \mathrm{e}$ com o número de fármacos prescritos. ${ }^{27,32,41-42}$ Neste estudo verifica-se mesmo significância estatística $(p=0,001)$ relativamente ao número de fármacos.

O conhecimento da finalidade da medicação prescrita e o facto de o utente preparar a sua própria medicação está associado a valores mais baixos de PMMajor e PIM (valores estatisticamente significativos), refletindo a importância da literacia em saúde.

A autoperceção positiva da saúde apresenta também significado estatístico em relação à PMMajor e PIM.

Este estudo pretende alertar para números relevantes de PM e risco de PIM. Pensamos que devem ser estabelecidos parâmetros de prescrição que tenham em conta os riscos potenciais, sobretudo na população mais idosa. É necessário estar alerta e criar o hábito de rever em todas as consultas toda a prescrição, quer sejam em situações agudas quer em consultas programadas, para reduzir o risco decorrente de iatrogenia.

\section{REFERÊNCIAS BIBLIOGRÁFICAS}

1. Instituto Nacional de Estatística. $O$ envelhecimento em Portugal: si- 
tuação demográfica e socio-económica recentes das pessoas idosas [Population ageing in Portugal: recent demographic and socio-economic situation of older persons]. Lisboa: INE; 2002. Portuguese

2. Instituto Nacional de Estatística. Censos 2011: resultados definitivos - Portugal. Lisboa: INE; 2012. ISBN 9789892501819

3. World Health Organization. Healthy ageing [Internet]. Euro.who.int [homepage]; 2017.Available from: http://www.euro.who.int/en/healthtopics/Life-stages/healthy-ageing/healthy-ageing

4. Paúl C, Ribeiro O. Manual de gerontologia: aspetos biocomportamentais, psicológicos e sociais do envelhecimento. Lisboa: LIDEL; 2012. ISBN 9789727577996

5. Organização Mundial da Saúde. Guia global: cidade amiga do idoso [Internet]. Genebra: OMS; 2008. ISBN 9789248547300. Available from: http://www.who.int/ageing/GuiaAFCPortuguese.pdf

6. Björkman IK, Fastbom J, Schmidt IK, Bernsten CB. Drug-drug interactions in the elderly. Ann Pharmacother. 2002;36(11):1675-81.

7. Bregnhøj L, Thirstrup S, Kristensen MB, Bjerrum L, Sonne J. Combined intervention programme reduces inappropriate prescribing in elderly patients exposed to polypharmacy in primary care. Eur J Clin Pharmacol. 2009;65(2):199-207.

8. Buurma H, Schalekamp T, Egberts AC, De Smet PA. Compliance with national guidelines for the management of drug-drug interactions in Dutch community pharmacies. Ann Pharmacother. 2007;41(12):2024-31.

9. Bjerrum L, Søgaard J, Hallas J, Kragstrup J. Polypharmacy in general practice: differences between practitioners. Br J Gen Pract. 1999;49(440): 195-8.

10. Silva P, Luís S, Biscaia A. Polimedicação: um estudo de prevalência nos centros de saúde do Lumiar e de Queluz [Polypharmacy: a prevalence study in the Lumiar and Queluz health centres]. Rev Port Clin Geral. 2004;20(3):323-36. Portuguese

11. Veehof L, Stewart R, Haaijer-Ruskamp F, Jong BM. The development of polypharmacy: a longitudinal study. Fam Pract. 2000;17(3):261-7.

12. Cima Cl, Freitas RS, Lamas MC, Mendes CA, Neves AC, Fonseca C. Consumo de medicação crónica: avaliação da prevalência no Norte de Portugal [Use of chronic medication: evaluation of the prevalence in northern Portugal]. Rev Port Clin Geral. 2011;27(1):20-7. Portuguese

13. Mizokami F, Koide Y, Noro T, Furuta K. Polypharmacy with common diseases in hospitalized elderly patients. Am J Geriatr Pharmacother. 2012;10(2):123-8.

14. Reid J, Crome, P. Polypharmacy: causes and effects in older people. Prescriber. 2005; 16(17):57-62.

15. Carneiro AV. O erro clínico, os efeitos adversos terapêuticos e a segurança dos doentes: uma análise baseada na evidência científica [Medical errors, therapeutic adverse effects and patient safety: an evidence-based analysis]. Rev Port Saúde Pública. 2010;10(temático):3-10. Portuguese

16. Gandhi TK, Weingart SN, Borus J, Seger AC, Peterson J, Burdick E, et al. Adverse drug events in ambulatory care. N Engl J Med. 2003;348(16): 1556-64.

17. World Health Organization. World alliance for patient safety - WHO draft guidelines for adverse event reporting and learning systems: from information to action [Internet]. Geneva:WHO; 2005. Available from: http://apps.who.int/iris/bitstream/10665/69797/1/WHO-EIP-SPOQPS-05.3-eng.pdf
18. Obreli Neto PR, Nobili A, de Lyra DP Jr, Pilger D, Guidoni CM, de Oliveira Baldoni $A$, et al. Incidence and predictors of adverse drug reactions caused by drug-drug interactions in elderly outpatients: a prospective cohort study. J Pharm Pharm Sci. 2012;15(2):332-43.

19. Mallet L, Spinewine A, Huang A. Prescribing in elderly people 2: the challenge of managing drug interactions in elderly people. Lancet. 2007;370(9582):185-91.

20. Beers MH, Baran RW, Frenia K. Drugs and the elderly, part 1: the problems facing managed care. Am J Manag Care. 2000;6(12):1313-20.

21. Beers $M H$, Ouslander JG. Risk factors in geriatric drug prescribing: a practical guide to avoiding problems. Drugs. 1989;37(1):105-12.

22. Fick DM, Cooper JW, Wade WE, Waller JL, Maclean JR, Beers MH. Updating the Beers criteria for potentially inappropriate medication use in older adults: results of a US consensus panel of experts. Arch Intern Med. 2003;163(22):2716-24.

23. Delgado-Silveira E, Muñoz-García MM, Montero-Errasquin B, SánchezCastellano C, Gallagher PF, Cruz-Jentoft AJ. Prescripción inapropiada de medicamentos en los pacientes mayores: los criterios STOPP/START [Inappropriate prescription in older patients: The STOPP/START criteria]. Rev Esp Geriatr Gerontol. 2009;44(5):273-9. Spanish

24. Soares MA, Fernandez-LLimós F, Lança C, Cabrita J, Morais JA. Operacionalização para Portugal: critérios de Beers de medicamentos inapropriados nos doentes idosos [Operationalization to Portugal: Beers criteria of inappropriate medication use in the elderly]. Acta Med Port. 2008;21(5):441-52. Portuguese

25. American Geriatrics Society 2012 Beers Criteria Update Expert Panel. American Geriatrics Society updated Beers Criteria for potentially inappropriate medication use in older adults. J Am Geriatr Soc. 2012;60 (4):616-31.

26. Fouquet A, Zegbeh H, Krolak-Salmon P, Mouchoux C. Detection of potentially inappropriate medication in a French geriatric teaching hospital: a comparison study of the French Beers criteria and the improved prescribing in the elderly tool. Eur Geriatr Med. 2012;3(5):326-9.

27. Fialová D, Topinková E, Gambassi G, Finne-Soveri H, Jónsson PV, Carpenter I, et al. Potentially inappropriate medication use among elderly home care patients in Europe. JAMA. 2005;293(11):1348-58.

28. Ferreira R. Consumo crónico de medicamentos na população de um centro de saúde [Chronic use of medications by the population of a health center]. Rev Port Clin Geral. 2007;23(2):125-32. Portuguese

29. Broeiro P, Ramos V. Patologia múltipla e polifarmácia no idoso. Rev Port Clin Geral. 1997;14(1):8-22.

30. Antunes P. Medicação crónica numa lista de utentes [Chronic medication in a patient list]. Rev Port Clin Geral. 2000;16(2):113-24. Portuguese

31. Vintém JM. Inquéritos nacionais de saúde - Auto-percepção do estado de saúde: uma análise em torno da questão de género e da escolaridade. Rev Saúde Publica. 2008;6(2):5-18.

32. Cannon KT, Choi MM, Zuniga MA. Potentially inappropriate medication use in elderly patients receiving home health care: a retrospective data analysis. Am J Geriatr Pharmacother. 2006;4(2):134-43.

33. Landi F, Russo A, Liperoti R, Barillaro C, Danese P, Pahor M, et al. Impact of inappropriate drug use on physical performance among a frail elderly population living in the community. Eur J Clin Pharmacol. 2007;63(8):791-9. 
34. Brekke M, Rognstad S, Straand J, Furu K, Gjelstad S, Bjørner T, et al. Pharmacologically inappropriate prescriptions for elderly patients in general practice: how common? Baseline data from The Prescription Peer Academic Detailing (Rx-PAD) study. Scand J Prim Health Care. 2008;26 (2):80-5.

35. Martins SO, Soares MA, Foppe Van Mil JW, Cabrita J. Inappropriate drug use by Portuguese elderly outpatients: effect of the Beers criteria update. Pharm World Sci. 2006;28(5):296-301.

36. Dedhiya SD, Hancock E, Craig BA, Doebbeling CC, Thomas J 3rd. Incident use and outcomes associated with potentially inappropriate medication use in older adults. Am J Geriatr Pharmacother. 2010;8 (6):562-70.

37. Akazawa M, Imai H, Igarashi A, Tsutani K. Potentially inappropriate medication use in elderly Japanese patients. Am J Geriatr Pharmacother. 2010;8(2):146-60.

38. Ray WA, Griffin MR, Schaffner W, Baugh DK, Melton LJ 3rd. Psychotropic drug use and the risk of hip fracture. N Engl J Med. 1987;316(7): 363-9.

39. Nurminen J, Puustinen J, Piirtola M, Vahlberg T, Kivelä SL. Psychotropic drugs and the risk of fractures in old age: a prospective population-based study. BMC Public Health. 2010;10:396.

40. Khong TP, de Vries F, Goldenberg JS, Klungel OH, Robinson NJ, Ibáñez
$L$, et al. Potential impact of benzodiazepine use on the rate of hip fractures in five large European countries and the United States. Calcif Tissue Int. 2012;91(1):24-31.

41. Johnell K, Klarin I. The relationship between number of drugs and potential drug-drug interactions in the elderly: a study of over 600,000 elderly patients from the Swedish Prescribed Drug Register. Drug Saf. 2007;30(10):911-8.

42. Shiyanbola OO, Farris KB. Concerns and beliefs about medicines and inappropriate medications: an Internet-based survey on risk factors for self-reported adverse drug events among older adults. Am J Geriatr Pharmacother. 2010;8(3):245-57.

\section{CONFLITO DE INTERESSES}

Os autores declaram não ter quaisquer conflitos de interesse.

\author{
ENDEREÇO PARA CORRESPONDÊNCIA \\ Márcia Mendonça Souto \\ E-mail:m2souto@hotmail.com
}

Recebido em 05-05-2017

Aceite para publicação em 01-02-2018

\section{ABSTRACT \\ CHRONIC THERAPY IN THE ELDERLY IN A FAMILY HEALTH UNIT: ANALYSIS OF POLYPHARMACY AND POTENTIALLY INAPPROPRIATE MEDICATION}

Introduction: The improvement of health care and socioeconomic conditions have contributed to an increased longevity of the population, which is associated with a higher prevalence of chronic diseases. This population often needs treatment by means of polypharmacy, which increases the risk of iatrogenic diseases. In elderly, drug interactions (DI) and exposure to the use of potentially inappropriate medication (PIM) can result in deleterious consequences for their health.

Objectives: This study aimed to better understand the use of chronic therapy by elderly aged 75 years old and over, registered at a Family Health Unit in Braga, Portugal. Specifically, we aimed to determine the frequency of polypharmacy and use of potentially inappropriate medication, and to identify predisposing factors associated to these problems.

Results: Amongst the 86 patients studied, $96.5 \%$ were chronic users of at least one medication. Twenty-four percent used 2-4 medications and $74.4 \%$ used five or more medications. In $37.7 \%$ of the patients, use of PIM was identified according to Beers criteria, with $90.7 \%$ of these cases exhibiting a 'high' degree of inadequacy. There was a statistical significant association between the number of medications used and PIM. These two variables were also statistically associated with 'self-perception of health', with 'who prepares the medication' and with 'knowledge of the purpose of medication'.

Discussion/Conclusion: Polypharmacy and PIM are extremely frequent amongst elderly aged 75 years or over, in the population studied. From the data obtained, the authors sought to have an understanding of the socioeconomic context associated with an increased risk, to help to inform a clinical strategy to reduce the risks of iatrogeny.

Keywords: Polypharmacy; Potentially inappropriate medication; Beers criteria. 